\title{
O PROCESSO DE TRABALHO DE ENFERMEIROS EM NÚCLEOS HOSPITALARES DE EPIDEMIOLOGIA*
}

Adeli Regina Prizybicien de Medeiros', Liliana Müller Larocca², Maria Marta Nolasco Chaves³, Aida Maris Peres ${ }^{4}$

'Enfermeira. Mestre em Enfermagem. Hospital de Clínicas da Universidade Federal do Paraná. Curitiba-PR- Brasil. ${ }^{2}$ Enfermeira. Doutora em Educação. Universidade Federal do Paraná. Curitiba-PR-Brasil. ${ }^{3}$ Enfermeira. Doutora em Ciências. Universidade Federal do Paraná. Curitiba-PR-Brasil. ${ }^{4}$ Enfermeira. Doutora em Enfermagem. Universidade Federal do Paraná. Curitiba-PR-Brasil.

RESUMO: Estudo exploratório e qualitativo, com o objetivo de caracterizar o processo de trabalho de enfermeiros de Núcleos Hospitalares de Epidemiologia quanto aos agentes, finalidade, meios e instrumentos, objeto e produtos. Os dados foram coletados, entre março e maio de 2012, por meio de entrevista aberta com nove enfermeiros de cinco Núcleos Hospitalares de Epidemiologia existentes em Curitiba-PR, em conformidade com a Resolução 196/96. O estudo permitiu identificar os elementos constituintes do trabalho do enfermeiro em Vigilância Epidemiológica Hospitalar e evidenciou práticas ainda incipientes em algumas instituições. Depreendeu-se que a prática profissional, nos referidos núcleos, deve romper com o modelo de atuação centrado em tarefas, para um modelo articulado com serviços de referência e que contribua com a construção de Políticas Públicas alinhadas às necessidades em saúde da população.

DESCRITORES: Enfermagem; Saúde coletiva; Vigilância epidemiológica.

\section{THE WORK PROCESS OF NURSES IN HOSPITAL EPIDEMIOLOGY CENTERS}

\begin{abstract}
This exploratory and qualitative study aimed to characterize the work process of nurses from Hospital Epidemiology Centers in relation to the agents, aim, means and instruments, object and products. The data were collected between March and May 2012, through open interviews held with nine nurses from five Hospital Epidemiology Centers in the city of Curitiba, Paraná, in accordance with Resolution 196/96. The study made it possible to identify the constituent elements of the work of the nurse in Hospital Epidemiological Surveillance and evidenced practices which are still incipient in some institutions. It emerged that the professional practice, in the centers referred to, must break from the taskcentered model of work, in favor of a model which is articulated with specialist services and which contributes to the construction of Public Policies aligned with the population's health needs.
\end{abstract}

DESCRIPTORS: Nursing; Collective health; Epidemiological surveillance.

\section{EL PROCESO DE TRABAJO DE ENFERMEROS EN NÚCLEOS HOSPITALARES DE EPIDEMIOLOGÍA}

RESUMEN: Estudio exploratorio y cualitativo, cuyo objetivo fue caracterizar el proceso de trabajo de enfermeros de Núcleos Hospitalares de Epidemiología acerca de los agentes, finalidad, médios, instrumentos, objeto y productos. Los datos fueron obtenidos entre marzo y mayo de 2012, por medio de entrevista abierta con nueve enfermeros de cinco Núcleos Hospitalares de Epidemiología de Curitiba-PR, de acuerdo a la Resolución 196/96. El estudio posibilitó identificar los elementos que hacen parte del trabajo del enfermeiro en Vigilancia Epidemiológica Hospitalar y evidenció prácticas todavía incipientes en algunas instituciones. Se constató que la práctica profesional, en los referidos núcleos, debe romper el modelo de actuación centrado en tareas, para un modelo articulado con servicios de referencia y que contribuya con la construcción de Políticas Públicas referentes a las necesidades en salud de la población.

DESCRIPTORES: Enfermería; Salud colectiva; Vigilancia epidemiológica.

*Artigo extraído da dissertação intitulada: Saberes Instrumentais e ideológicos de enfermeiros no Processo de Trabalho em Vigilância Epidemiológica Hospitalar. Universidade Federal do Paraná, 2012.

Autor Correspondente:

Adeli Regina Prizybicien de Medeiros

Hospital de Clínicas da Universidade Federal do Paraná

Rua General Carneiro, 181 - 80060-120 - Curitiba-PR-Brasil

E-mail: adeli.medeiros@ufpr.br
Recebido: 05/05/2014

Finalizado: 13/11/2014 


\section{INTRODUÇÃO}

O Subsistema Nacional de Vigilância Epidemiológica em âmbito hospitalar foi instituído pelo Ministério da Saúde com a Portaria n. ${ }^{\circ}$ $2529 / 2004^{(1-2)}$, a partir do reconhecimento do caráter articulador e fomentador de ações de prevenção e controle de agravos representado pelas instituições hospitalares. Atualmente, no Brasil, 190 hospitais compõem a Rede de Hospitais de Referência Nacional, sendo dez no estado do Paraná, dos quais cinco estão localizados na cidade de Curitiba, $\mathrm{PR}^{(3)}$. As constatações oriundas da prática profissional sobre a significativa inserção de enfermeiros nos Núcleos Hospitalares de Epidemiologia (NHE), a peculiaridade dos diferentes cenários hospitalares e o caráter heterogêneo das ações dos enfermeiros atuantes em Vigilância Epidemiológica Hospitalar (VEH) motivaram a realização de um projeto de mestrado em Prática Profissional de Enfermagem ${ }^{(4)}$, o qual teve por objeto de estudo os saberes que subsidiavam os processos de trabalho dos enfermeiros nos NHE de Curitiba, PR, sendo este artigo parte do referido projeto.

A Vigilância Epidemiológica Hospitalar se constitui um campo profícuo de atuação, gerando desafios e inúmeras possibilidades de investigação. O estado da arte na área se concentra em investigações de surtos e proposição de condutas para o controle de agravos específicos na Atenção Básica, mas é restrito no apontamento de referenciais teóricometodológicos sobre o processo de trabalho do enfermeiro $^{(5)}$. Nesse sentido, o objetivo deste estudo foi caracterizar o processo de trabalho de enfermeiros de NHE quanto aos agentes, finalidade, meios e instrumentos, objeto e produtos.

\section{MÉTODO}

O estudo teve abordagem qualitativa e cunho exploratório e a coleta de dados foi realizada, no período de março a maio de 2012, por meio de entrevista aberta, gravada, com todos os enfermeiros que atuavam nos cinco núcleos hospitalares de epidemiologia existentes na cidade de Curitiba (PR), num total de nove profissionais. Após leitura e assinatura do Termo de Consentimento e Livre e Esclarecido foi solicitado aos sujeitos a descrição de uma semana típica de trabalho e (ou) o relato de situações inusitadas vivenciadas, nas quais tivessem se apropriado da Epidemiologia como base teórica, para enfrentamento do trabalho cotidiano. Para garantia de anonimato, os dados coletados mediante entrevistas não foram identificados e sim codificados de forma aleatória com a letra E seguida do numeral ordinal de 1 a 9 (E1 a E9), independente da instituição de vínculo.

O agrupamento inicial das falas foi por instituição, de modo a facilitar a transcrição e compreensão dos contextos em que foram proferidos. A seguir, após leitura exaustiva, foram identificadas frases temáticas presentes em cada um dos discursos, a partir das quais foram construídas duas categorias empíricas com intenção classificatória( ${ }^{(6)}$ : Saberes Instrumentais e Saberes Ideológicos ${ }^{(4)}$. A finalidade foi abranger conceitos semelhantes e abrir caminho à Análise do Discurso ${ }^{(7)}$, possibilitando a caracterização dos agentes, finalidade, meios e instrumentos, objeto e produtos inerentes ao processo de trabalho de enfermeiros de NHE.

O projeto foi aprovado pelo Comitê de Ética do Setor de Ciências da Saúde da Universidade Federal do Paraná (UFPR) sob o n. ${ }^{\circ}$ 0110.0.091.09111. Foram atendidas as diretrizes da Resolução 196/96.

\section{RESULTADOS}

A análise dos discursos evidenciou os elementos constitutivos do processo de trabalho de enfermeiros em VEH. Foi notória a importância atribuída pelos entrevistados à organização do deste processo em VEH, já que em todos os discursos observaram-se aspectos relacionados à preocupação em implantar e organizar rotinas, buscar visibilidade institucional e desenvolver ações articuladas à confirmação diagnóstica. Os discursos ratificaram volume significativo de atividades diárias dos enfermeiros, fragmentadas e compostas de diversas e repetitivas etapas.

Sobre os Agentes - constatou-se que, dos nove enfermeiros entrevistados, quatro trabalhavam em três hospitais que prestavam serviços exclusivamente ao SUS e cinco distribuídos em outros dois hospitais que, além do SUS, 
atendiam a pacientes com algum plano de seguridade privada. Entre os nove enfermeiros, cinco apresentaram idades entre 20 e 30 anos e atuavam na área de VEH em períodos que variaram de menos de um ano a três anos. Entre os demais, quatro referiram idade maior que 30 anos e atuações em núcleos hospitalares de epidemiologia há mais de três anos.

Em relação ao treinamento específico para a atuação nos NHE, apenas dois profissionais referiram capacitação em Vigilância Epidemiológica, na forma de cursos e oficinas promovidos pelas esferas municipal, estadual e federal de governo. Entre os demais houve menção ao preparo para as funções, por meio da experiência de colegas da instituição; experiência assistencial em hematologia como preponderante para o bom desempenho no Registro Hospitalar de Câncer, uma das atividades do NHE; a participação em reuniões nas Secretarias Municipal e Estadual de Saúde e o desenvolvimento de estágio curricular/elaboração de monografia na área.

Dois enfermeiros ressaltaram quanto o preparo técnico adequado é necessário para as ações do $\mathrm{NHE}$, especialmente, na vigência de situações inusitadas que requerem ações imediatas, no cotidiano da vigilância epidemiológica hospitalar.

Quanto à relação entre o Serviço de Controle de Infecção (SCIH) e o NEH constatou-se que, em três instituições, os dois serviços compartilhavam o mesmo espaço e divergiam tanto nas nomenclaturas para a forma como o Serviço era conhecido, quanto ao grau de entrosamento para o desenvolvimento das ações de Controle de Infecção Hospitalar e Vigilância das doenças de notificação compulsória.

Sobre as Finalidades, os discursos dos enfermeiros evidenciaram o caráter de vigilância, promoção da saúde, prevenção e controle de agravos e doenças de notificação compulsória em âmbito hospitalar.

Entre os Meios e Instrumentos constitutivos do processo de trabalho em VEH, os discursos denotaram a importância do arsenal legislativo e normativo, bem como o papel das diferentes fichas de notificação e de formulários, para a realização da busca ativa de doenças de notificação compulsória pelo NEH.

Dentre o conjunto de ações desenvolvidas em âmbito hospitalar - Objeto do Processo de
Trabalho dos enfermeiros em VEH - destacou-se a busca ativa de doenças e agravos. Dos nove enfermeiros, à exceção de um, exclusivo do Registro Hospitalar de Câncer, todos referiram dedicar-se à busca diária de doenças de notificação compulsória entre pacientes atendidos em pronto atendimento, pronto socorro e internação.

Em adição, em sete discursos foram referidos o preenchimento das fichas de notificação; identificação de amostras laboratoriais já coletadas e orientação sobre coletas a serem realizadas, para posterior envio ao Laboratório Central do Estado (Lacen) e registro das amostras, quando o laboratório não se encarregava disso. Em geral, os resultados destes exames subsidiavam o encerramento das fichas de notificação pelos enfermeiros.

Quatro enfermeiros associaram a visibilidade das ações de Vigilância Epidemiológica com a obtenção de espaços para discussão de assuntos desta natureza, na forma de reuniões com equipes, serviços, departamentos e, inclusive, com as diretorias de cada instituição. Entretanto, em dois discursos houve indicação de maior ênfase à discussão de assuntos de Controle de Infecção:

Para novos colaboradores a gente faz a integração e essa aula é muito rápida e muito pobre, destinada a diversas categorias profissionais, [...] Falo sobre composição da equipe, Epidemiologia, CCIH e Gerenciamento de Riscos. [...] Sobre a Epidemiologia eu dou um apanhado geral do que fazer[...] reportar os agravos de notificação para a Secretaria Municipal de Saúde [...] daí a gente acaba citando os agravos de notificação [...]. (E8)

O trabalho articulado de busca ativa da Vigilância Epidemiológica para a captação também de casos de infecção hospitalar foi referido por apenas um dos sujeitos. No discurso de seis participantes houve tentativas de estabelecimento de diferenças entre o trabalho de Vigilância Epidemiológica Hospitalar e o de Controle de Infecção Hospitalar:

O SCIH é bem mais antigo, o Núcleo de Epidemiologia veio depois, em 2006., sempre tem a confusão com o $\mathrm{SCIH},[\ldots]$ a gente sempre faz questão de esclarecer a diferença, 
principalmente porque a gente encabeça as campanhas de vacinação[...]. (E4)

A percepção dos sujeitos sobre seu próprio trabalho evidenciou singularidades em cinco discursos. Nessas falas emergiram questões acerca da associação, por alguns colegas assistenciais e pelo meio acadêmico, que destacavam as atividades de Vigilância Epidemiológica e Controle de Infecção como de menor complexidade:

[...] acho que as equipes não compreendem as atividades do Núcleo de Epidemiologia [...] nos vêem como profissionais que preenchem papel para mandar para o município [...]. (E8)

[...] extremamente complicado, minucioso [...], até o próprio pessoal da graduação tem aquela visão que é enfermeiro administrativo, que não tem muito trabalho, mas se assustam com o nivel de complexidade das nossas atividades. (E4)

A atuação em comissões e comitês foi identificada em três discursos, desde a preparação de casos até a organização de reuniões para discussão. Ainda, atribuições como as relacionadas à orientação e administração de vacinas em ambiente hospitalar, tanto para pacientes quanto para colaboradores, integraram o conjunto de atividades desempenhadas pelos enfermeiros.

Finalmente, os enfermeiros referiram em seus discursos à importância dos produtos da Vigilância Epidemiológica Hospitalar no controle de surtos e epidemias, na instituição e para além do ambiente hospitalar, entre eles: Saberes para a intervenção junto ao usuário; Saberes para a intervenção junto à sua família; Saberes para a intervenção junto a serviços e equipes do hospital; Saberes para intervenção junto a grupos populacionais e Saberes para a intervenção interinstitucional.

Os saberes para a intervenção junto aos usuários e suas famílias foram mencionados por três enfermeiros ao se referirem a realização de orientações, indicação de bloqueio medicamentoso, nos casos de meningite e coqueluche, bem como para a coleta pelos próprios enfermeiros do Núcleo das amostras laboratoriais de contatos familiares:
[...] às vezes é a mãe que está tossindo, então a gente coleta da criança e da mãe, cadastra no sistema e manda para o LACEN. (E3)

Entre os saberes para a intervenção junto a serviços e equipes do hospital, em cinco discursos os enfermeiros ressaltaram seu papel formativo e de orientação, em virtude da inserção do Núcleo Hospitalar de Epidemiologia em hospital universitário, em um cenário em que todos os hospitais têm atividades de ensino:

[...] Onosso papel aqui como hospital universitário é educar, nosso papel é ensinar [...] a gente faz o máximo esforço quando os residentes entram [...] estamos aqui para que tenham esse conhecimento das necessidades que uma doença de notificação exige [...]. (E4)

A divulgação dos dados notificados apresentase como um saber originado a partir das notificações epidemiológicas, que após as devidas análises na instituição, pode vir a constituir informações úteis à instituição e à comunidade. Mesmo que a divulgação dos dados ainda não fosse uma constante na opinião dos enfermeiros, existia o reconhecimento da importância dos dados gerados pelo Núcleo de Epidemiologia como possibilidade de divulgação junto ao meio científico e para a comunidade, como nesse discurso:

[...] nossa chefia propôs para o Departamento de Marketing de se criar um boletim como já existe em outros hospitais, divulgado para todos. Mas tudo isso depende de custo, de aprovação, sem certeza de liberação. (E8)

No produto relacionado a saberes para a intervenção junto a grupos populacionais houve destaque o Registro Hospitalar de Câncer. Nele, o volume e a complexidade das atividades de registro foram aparentes em quatro discursos, com ênfase para suas possibilidades de contribuição em prol da melhoria da qualidade assistencial, a partir da análise e divulgação dos dados de câncer:

[...] Gosto deste trabalho por contribuir com o INCA [Instituto Nacional do Câncer] e com o 
hospital, eu me identifiquei com a investigação [...] Se não fosse a contribuição do Registro de Câncer com esses dados não se podia fazer campanhas, porque se você não tiver o dado em mãos, como é que você vai subsidiar informações para campanhas [...] eu penso que a informação é a base para você poder tratar. (E7)

Entre os saberes para a intervenção interinstitucional observou-se a articulação do trabalho de VEH com as demais instâncias da rede de assistência à saúde. Em cinco discursos os enfermeiros referiram priorizar o acionamento do distrito sanitário nos casos de doenças que requeriam o desencadeamento de ações de bloqueio aos familiares dos casos índice, bem como dos contatos comunitários:

[...] quando a gente tem todas as informações, a gente volta, liga e passa as informações para o distrito poder avisar o próximo distrito ou a outra regional de saúde, agilizamos esse processo em casos de alta. (E3)

Nessa relação, o termo "ponte" foi utilizado em três discursos para ilustrar o papel do Núcleo de Epidemiologia com a Secretaria Municipal deSaúde, por meio da relação de parceria com o distrito.

Entretanto, foi identificada certa alienação do seu próprio trabalho, bem como descrença dos desdobramentos de suas ações na articulação com o Distrito Sanitário. O discurso do enfermeiro E9 afirma:

[...] me sinto assim como uma fornecedora de informação! Você se vira do avesso para conseguir as coisas e depois não fica sabendo o que aconteceu [...] uma coisa que eu particularmente sinto bastante falta é de ter um retorno específico do Distrito sobre o que foi notificado. $O$ Distrito tem os relatórios dele, mas não informa as medidas que foram tomadas, eu não sei se eles vão dar continuidade naquilo que comecei. (E9)

Esse discurso, ao se referir à articulação do Núcleo Hospitalar de Epidemiologia com o Distrito Sanitário, foi único e constituiu um dos exemplos das barreiras que a VEH enfrenta para seu reconhecimento tanto hospitalar, quanto nas demais esferas do sistema de saúde.

\section{DISCUSSÃO}

Considerando que a Vigilância Epidemiológica das Doenças de Notificação Compulsória em âmbito hospitalar é um campo de atuação profissional recente, instituído em 2004(1), observou-se consonância entre a recente implantação dos serviços e a preocupação dos profissionais em organizar seus processos de trabalho. Em comparação com o Relatório Estadual $^{(3)}$ do ano de 2008 manteve-se a maior presença de enfermeiros, como força de trabalho nos Núcleos Hospitalares de Epidemiologia, desenvolvendo grande parte das atividades realizadas nos cenários, independente do caráter interdisciplinar do trabalho executado.

Da mesma forma, em conformidade com o mesmo relatório, persiste o reduzido quantitativo de profissionais com preparo técnico específico, a partir de Cursos Básicos de Vigilância Epidemiológica e (ou) treinamento para realização de análise de dados ${ }^{(3)}$.

A maior concentração de profissionais em hospitais que prestam atendimento exclusivo ao SUS permitiu considerar que a Vigilância Epidemiológica está profundamente ligada as políticas públicas de saúde ${ }^{(1)}$, visto que um dos critérios para inclusão no subsistema e recebimento de repasse financeiro mensal foi a prestação de serviços ao SUS.

O grande volume de atividades diárias assumidas pelos enfermeiros destacou um processo de trabalho tecnicista e fragmentado, com uma visão biologicista do ser humano ${ }^{(8)}$. Em concordância com outros autores ${ }^{(9)}$, a falta de clareza do trabalhador sobre os elementos constituintes deste processo foi apontada como uma das possíveis causas de alienação sobre seus produtos. O conhecimento e reflexão sobre seus elementos constituintes pode contribuir para a detecção de possíveis lacunas e para a melhor delimitação de suas funções ${ }^{(10)}$ no processo de trabalho em VEH.

Conquanto, observou-se consonância entre o arsenal legislativo que regulamenta o Subsistema Nacional de Vigilância Epidemiológica, as finalidades do processo de trabalho e as atividades desenvolvidas pelos participantes do estudo, em cada um dos cenários estudados ${ }^{(1-3)}$.

Acerca dos meios e instrumentos utilizados foi notória a associação entre os conhecimentos 
técnicos inerentes à profissão, as técnicas e os relacionamentos para atuação junto às equipes no dia-a-dia de vigilância epidemiológica desenvolvida nos hospitais. Em três discursos foram referidas abordagens pautadas em orientação, recomendação técnica, retorno de informações e o contato pessoal com as equipes assistenciais como importantes propulsoras de uma visibilidade positiva das atividades do Núcleo de Epidemiologia.

A busca ativa de doenças e agravos teve destaque para a concretização do Objeto do Processo de Trabalho - ações de Vigilância Epidemiológica desenvolvidas em âmbito hospitalar. A despeito do discurso de seis participantes, o trabalho em conjunto do $\mathrm{SCIH} \mathrm{e}$ do Núcleo de Epidemiologia evitaria superposição de trabalhos e traria contribuições ao processo de trabalho ${ }^{(11)}$. Nesta pesquisa, em três hospitais, o Núcleo de Epidemiologia está vinculado ao $\mathrm{SCIH}$.

A percepção pelos outros profissionais dos hospitais sobre as atividades realizadas na VEH, tidas como trabalho "administrativo, fácil e burocrático" mostrou-se contrária ao que alguns sujeitos pensam do próprio trabalho, descrito como minucioso e complicado. A partir dos discursos depreendeu-se a necessidade de maior atenção a ações de divulgação dos dados, constatada anteriormente, pela Secretaria Estadual de Saúde ${ }^{(3)}$.

Como forma de superação, autoras ${ }^{(11-12)}$ afirmam que a divulgação de boletins e campanhas informativas, realização de treinamentos e a melhor integração do NHE com serviços, gerências e direções podem representar maior legitimidade, aporte de recursos e ampliação do papel de VEH, inclusive pela maior sensibilização dos profissionais das instituições.

Os Produtos da VEH corroboram o papel de referência dos Núcleos no fornecimento de informações epidemiológicas dos adoecimentos ou de mortalidade ${ }^{(11-12)}$. Em adição, contribuem para o aumento das notificações espontâneas pelos profissionais da instituição, bem como para o conhecimento e a valorização do papel de VEH articulado com a Atenção Básica ${ }^{(13)}$. A comunicação de agravos e doenças ao Distrito Sanitário e deste para as outras instâncias da rede de atenção à saúde foi caracterizada como via de mão dupla, em conformidade com a legislação federal ${ }^{(13)}$.
Entretanto, sobre a dissociação e falta de retroalimentação entre as atividades de VEH e as de vigilância à saúde realizadas no cenário da Atenção Básica, acredita-se que tal realidade seja proveniente da não superação do modelo assistencial curativo de Saúde Pública, que não reconhece ações de VE como parte do processo assistencial e sim como algo externo ao processo de trabalho desenvolvido em instituições hospitalares ${ }^{(10)}$.

Tal constatação corrobora a importância de utilização da Epidemiologia como importante ferramenta para a interpretação da realidade e a proposição de práticas profissionais frente aos modelos políticos e de saúde vigentes ${ }^{(14)}$.

\section{CONCLUSÕES}

Este estudo é uma contribuição à consolidação do trabalho do enfermeiro na Vigilância Epidemiológica em âmbito hospitalar, seja por caracterizar os elementos constituintes do processo de trabalho em diferentes cenários, como por colaborar na valorização profissional. A importância que os entrevistados atribuíram à organização do processo de trabalho ilustrou o longo caminho a ser trilhado, até a construção de um histórico institucional em VEH e a aquisição de experiência dos enfermeiros na área.

A prática profissional deve primar pela melhoria da integração entre a Atenção Básica e a Assistência Hospitalar, em consonância com o papel de "ponte" muito presente nos discursos dos enfermeiros dos NHE. Ainda, deve romper com o modelo de atuação centrado em tarefas, para um modelo que contribua com a construção de estratégias que possibilitem a concepção de Políticas Públicas que atendam as reais necessidades de saúde da população.

A superação de modelos de saúde curativistas e focados em indivíduos será possível com a retroalimentação de informações e com a interinstitucionalidade das ações, em um cenário em que indivíduos não sejam números e sim sujeitos numa dada realidade, com particularidades institucionais e singularidades reconhecidas. É a partir do fortalecimento das ações interinstitucionais, junto a grupos prioritários, que se pode contribuir na diminuição das desigualdades sociais e na melhoria das 
condições de saúde dos cidadãos brasileiros.

Destarte, ao caracterizar o processo de trabalho de enfermeiros de NHE, este estudo permitiu dar visibilidade ao importante espaço de atuação dos enfermeiros, contribuindo, assim, à Enfermagem como ciência.

\section{REFERÊNCIAS}

1. Ministério da Saúde. Portaria n. 2.529, de 23 de novembro de 2004: institui o Subsistema Nacional de Vigilância Epidemiológica em Âmbito Hospitalar. Diário Oficial da União, Brasília (DF) de 23 nov 2004.

2. Siqueira Filha NT, Vanderlei LCM, Mendes MFM. Avaliação do subsistema nacional de vigilância epidemiológica em âmbito hospitalar no estado de Pernambuco, Brasil. Epidemiol. Serv. Saúde [Internet] 2011;20(3) [acesso em: 28 out 2014]. Disponível: http://scielo.iec.pa.gov.br/scielo.php?script=sci_ arttext\&pid=S1679-49742011000300005\&lng=pt

3. SESA - Secretaria de Estado da Saúde. Relatório dos núcleos hospitalares de epidemiologia/NHE Paraná; [Internet] 2008 [acesso em: 25 out 2014]. Disponível: http://www.saude.pr.gov.br/arquivos/File/CIB/ATA/ ATA04/Relatorio_NHE_CIB.pdf

4. Medeiros ARP. Saberes instrumentais e ideológicos de enfermeiros no processo de trabalho em vigilância epidemiológica hospitalar [dissertação]. Curitiba (PR): Universidade Federal do Paraná; 2012.

5. Passos LMR. Assistir e vigiar: as ações da vigilância epidemiológica na unidade básica de saúde: situação atual e perspectivas [tese]. Ribeirão Preto (SP): Universidade de São Paulo; 2003.

6. Deslandes SF, Minayo MCS, organizadora. Pesquisa social: teoria, método e criatividade. $32^{\mathrm{a}}$ ed. Petrópolis: Vozes; 2012.

7. Fiorin, JL. Elementos da análise do discurso. $15^{\mathrm{a}}$ ed. São Paulo: Contexto; 2013.

8. Mendes-Gonçalves, R.B. Práticas de saúde: processos de trabalho e necessidades. São Paulo: Cefor; 1992.

9. Egry, EY. Saúde Coletiva: construindo um novo método em Enfermagem. São Paulo: Ícone; 1996.

10. Thofehrn MB, Montesinos MJL, Arrieira IC, Àvila AV, Vasques TCS, Farias ID. Processo de trabalho dos enfermeiros de um hospital da Espanha: ênfase nas tecnologias de cuidado. Cogitare enferm. [Internet] 2014;19(1) [Acesso em: 10 abr 2014]. Disponível: http://ojs.c3sl.ufpr.br/ojs/index.php/cogitare/article/ view/35972
11. Schettert PAS. Vigilância hospitalar: possibilidades e obstáculos de uma prática integrada [tese]. Rio de Janeiro (RJ): Instituto de Medicina Social; 2008.

12. Secretaria de Estado da Saúde de São Paulo. Vigilância epidemiológica em âmbito hospitalar. Rev. De Saúde Pública [Internet] 2007;41(3) [Acesso em: 25 out 2014] Disponível: http://www.scielo.br/pdf/rsp/v41n3/ itsaude.pdf

13. Ministério da Saúde. Portaria n. 2.254 de 05 de agosto de 2010: institui a Vigilância Epidemiológica em Âmbito Hospitalar e dá outras providências. Diário Oficial da União, 05 ago 2010.

14. Medeiros ARP, Larocca LM, Chaves MMN, Meier MJ, Wall ML. A epidemiologia como referencial teóricometodológico no processo de trabalho do enfermeiro. Rev. Esc. Enferm. USP. 2012;46(6):1519-23. 